\title{
Duality of positive and negative integrable hierarchies via relativistically invariant fields
}

\author{
S.Y. Lou, ${ }^{a, 1}$ X.B. Hu $\mathbf{u}^{b, c}$ and Q.P. $\mathbf{L i u}^{d}$ \\ ${ }^{a}$ School of Physical Science and Technology, Ningbo University, \\ Ningbo, 315211, China \\ ${ }^{b}$ LSEC, ICMSEC, Academy of Mathematics and Systems Science, Chinese Academy of Sciences, \\ Beijing 100190, China \\ ${ }^{c}$ School of Mathematical Sciences, University of Chinese Academy of Sciences, \\ Beijing 100049, China \\ ${ }^{d}$ Department of Mathematics, China University of Mining and Technology, \\ Beijing 100083, China \\ E-mail: lousenyue@nbu.edu.cn, hxb@lsec.cc.ac.cn, qpl@cumtb.edu.cn
}

ABSTRACT: It is shown that the relativistic invariance plays a key role in the study of integrable systems. Using the relativistically invariant sine-Gordon equation, the Tzitzeica equation, the Toda fields and the second heavenly equation as dual relations, some continuous and discrete integrable positive hierarchies such as the potential modified Kortewegde Vries hierarchy, the potential Fordy-Gibbons hierarchies, the potential dispersionless Kadomtsev-Petviashvili-like (dKPL) hierarchy, the differential-difference dKPL hierarchy and the second heavenly hierarchies are converted to the integrable negative hierarchies including the sG hierarchy and the Tzitzeica hierarchy, the two-dimensional dispersionless Toda hierarchy, the two-dimensional Toda hierarchies and negative heavenly hierarchy. In $(1+1)$-dimensional cases the positive/negative hierarchy dualities are guaranteed by the dualities between the recursion operators and their inverses. In $(2+1)$-dimensional cases, the positive/negative hierarchy dualities are explicitly shown by using the formal series symmetry approach, the mastersymmetry method and the relativistic invariance of the duality relations. For the 4-dimensional heavenly system, the duality problem is studied firstly by formal series symmetry approach. Two elegant commuting recursion operators of the heavenly equation appear naturally from the formal series symmetry approach so that the duality problem can also be studied by means of the recursion operators.

KEYworDS: Integrable Hierarchies, Integrable Field Theories, Space-Time Symmetries

ARXIV EPRINT: 2103.04549

\footnotetext{
${ }^{1}$ Corresponding author. Data Availability Statement: The data that support the findings of this study are available from the corresponding author upon reasonable request.
} 


\section{Contents}

1 Introduction 1

2 Duality of the PMKdV hierarchy and the sine-Gordon hierarchy 3

3 Duality of the PFG hierarchy and the Tzitzeica hierarchy 6

4 Duality of the dKPL hierarchy and the two-dimensional dispersionless $\begin{array}{ll}\text { Toda hierarchy } & 8\end{array}$

5 Duality hierarchies from the two-dimensional Toda lattice 11

6 From the real second heavenly equation to dual hierarchies 12

$\begin{array}{lll}7 & \text { Summary and discussions } & 14\end{array}$

\section{Introduction}

The study on dualities such as the wave-particle duality in quantum physics [1], the electromagnetic duality between electric and magnetic fields [2], AdS/CFT duality (duality between the Anti-de Sitter gravity and the conformal field theory) [3], the particle-vortex duality in condensed matter physics [4], the boson/fermion dualities in particle physics [5, 6] and quantum/classical duality between quantum and classical integrable fields [7] is an important topic in Physics. In this paper, we study the positive/negative hierarchy dualities, the dualities between integrable positive hierarchies and negative hierarchies by means of the relativistically invariant fields, such as the sine-Gordon (sG) field, the Tzitzeica field, the Toda fields and the heavenly equations.

In (1+1)-dimensional cases, the positive/negative hierarchy dualities are equivalent to the local/nonlocal symmetry dualities for the related integrable systems. The investigation of symmetries plays a fundamental role in natural science. It is known that the standard model including all the known elementary particles constituted our universe is based on the local $\mathrm{SU}(3) \times \mathrm{SU}(2) \times \mathrm{U}(1)$ gauge symmetry [8-10]. All the predictions resulted from the standard model have been confirmed whence the Higgs boson is found $[11,12]$. In nonlinear science, there are numerous models to describe real natural phenomena. While there is no unified method to solve nonlinear systems, the symmetry approach has been very effective. Symmetries have numerous applications [13], including to build new solutions from known ones $[14,15]$, to do dimensional reductions of nonlinear partial differential equations [1619], to get new integrable systems [20-23] and even to construct all solutions for certain nonlinear systems [24]. 
For a (1+1)-dimensional integrable system, there are infinitely many local and nonlocal symmetries. To produce a hierarchy of infinitely many local commuting symmetries, one can apply a recursion operator [25], $\Phi$, to a seed symmetry which usually lies in a kernel of the inverse recursion operator $\Phi^{-1}$. To find a set of infinitely many nonlocal symmetries, some different approaches are available. The simplest way is to apply the inverse of recursion operator, $\Phi^{-1}$, to a seed symmetry which belongs to a kernel of the recursion operator $\Phi[26]$. In addition to the kernels of the recursion operators, there are many other ways to find seed nonlocal symmetries, say, the squared eigenfunction symmetries [27] related to Lax pairs, the residual symmetries of the truncated Painlevé expansions [6], the infinitesimal Darboux transformations [23, 28, 29], the infinitesimal Bäcklund transformations [30, 31] and the infinitesimal conformal transformations of the Schwarzian forms [32].

For a $(2+1)$-dimensional integrable model, it is much more difficult to find symmetries because the nonexistence of recursion operator except for C-integrable models (the models which can be directly linearized) and breaking soliton models (whose recursion operators are of one-dimensional). To find the symmetries related to $(2+1)$-dimensional positive hierarchies, the mastersymmetry method [33] and the formal series symmetry approach (FSSA) [34] are two effective methods. To find (2+1)-dimensional negative integrable hierarchies, one may apply the formal spectral parameter expansion method [23, 30, 35] to suitable seed nonlocal symmetries like the squared eigenfunction symmetries, residual symmetries and infinitesimal Darboux/Bäcklund transformations.

To describe the colourful real nonlinear natural world, there are various idealized models including the Korteweg-de Vries (KdV) equation [36, 37], the modified KdV (MKdV) equation [38], the potential KdV (PKdV) equation, the potential MKdV (PMKdV) equation, the sine-Gordon (sG) equation [39], the Sawada-Kotera (SK) equation [40, 41], the Kaup-Kupershmidt (KK) equation [42, 43], the Fordy-Gibbons (FG) equation [44], the potential FG (PFG) equation, the Tzitzeica equation [45-48], the nonlinear Schrödinger (NLS) equation [49] and so on. It is interesting to point out that some of these physically relevant models are linked each other. Indeed, the MKdV equation is related to the $\mathrm{KdV}$ equation by a Miura transformation. The PMKdV equation is a potential form of the MKdV equation. The SK equation and the KK equation are linked to a common modified equation, the FG equation, via different Miura transformations [44]. The KdV equation, the MKdV equation, the NLS equation and the sG equation are all the special reductions of the Ablowitz-Kaup-Newell-Segur hierarchy $[50,51]$. The PMKdV equation and the sG equation possess a same recursion operator and some sets of common infinitely many conserved invariants and symmetries. The PFG equation and the Tzitzeica equation share a common recursion operator and some sets of invariants and symmetries. The sG equation and the Tzitzeica equation are all relativistically invariant, invariant under the Lorenz transformation in the experimental coordinate system or invariant under the space-time exchange in the light cone coordinate. In this paper, we study another type of connection among these integrable systems by means of the relativistically invariant equations like the sG, Tzitzeica, Toda and heavenly equations.

The paper is organized as follows. In section 2, with the help of the relativistic invariance of the sG equation, we study the local/nonlocal symmetry duality or equivalently the 
positive/negative hierarchy duality related to the PMKdV equation and the sG equation, i.e., the duality of the PMKdV hierarchy and the sG hierarchy. The duality of the PFG hierarchies and the Tzitzeica hierarchies are established in section 3 thanks to the relativistic invariance of the Tzitzeica equation. In section 4, after reviewing the FSSA and then applying the method to a $(2+1)$-dimensional potential dKPL model, the positive/negative hierarchy duality is established by using the two-dimensional dispersionless Toda (2ddT) equation. In section 5, the duality method is treated alternatively. We directly use the relativistically invariant differential-difference Toda equation to find a higher order Toda system and its related dual model. In section 6 , the hierarchy duality problem is studied for a four dimensional integrable model (the second heavenly equation) by means of the FSSA. Two commute elegant recursion operators of the second heavenly equation are reobtained from the FSSA. Then, the positive/negative heavenly hierarchy duality is established with the helps of the recursion operators and the relativistic invariance of the heavenly equation. The last section is a short summary and some discussions.

\section{Duality of the PMKdV hierarchy and the sine-Gordon hierarchy}

The sG equation (in the light cone coordinate)

$$
v_{x \tau}=\sin (v)
$$

is one of the most physically relevant field equations [39, 52-57]. It is important not only in quantum and classical field theories but also in almost all the physical branches and even in other natural scientific fields. For instance, the sG model is equivalent to the massive Thirring model [58, 59], the two-dimensional Coulomb gas $[54,60]$, the continuous limit of lattice $x-y-z$ spin-half model [61], and the massive $O(2)$ nonlinear $\sigma$ model [54].

It is known that the sG model (2.1) and the PMKdV equation

$$
v_{t}=v_{x x x}+\frac{1}{2} v_{x}^{3}
$$

share a common recursion operator

$$
\Phi=\partial_{x}^{2}+v_{x}^{2}-v_{x} \partial_{x}^{-1} v_{x x}
$$

where $\partial_{x}^{-1}$ is formally defined by $\partial_{x}^{-1} \partial_{x}=\partial_{x} \partial_{x}^{-1}=1$. When the operator, $\partial_{x}^{-1}$, is restricted to act on the functions with vanishing boundary at $x=-\infty$, one can simply define it by $\partial_{x}^{-1}=\int_{-\infty}^{x} \mathrm{~d} y[62]$.

Usually, the inverse recursion operator of $\Phi$ is written as [26, 62, 63]

$$
\Phi^{-1}=\partial_{\sin }^{-2}+\partial_{\cos }^{-2}=\frac{1}{2}\left(\partial_{\mathrm{e}^{i v}}^{-1} \partial_{\mathrm{e}^{-i v}}^{-1}+\partial_{\mathrm{e}^{-i v}}^{-1} \partial_{\mathrm{e}^{i v}}^{-1}\right),
$$

where the operators $\partial_{\sin }^{-1}, \partial_{\cos }^{-1}, \partial_{\mathrm{e}^{i v}}^{-1}$ and $\partial_{\mathrm{e}^{-i v}}^{-1}$ are defined by

$$
\partial_{\sin }^{-1} f \equiv \partial_{x}^{-1}[\sin (v) f], \partial_{\cos }^{-1} f \equiv \partial_{x}^{-1}[\cos (v) f], \partial_{\mathrm{e}^{\mathrm{i} v}}^{-1} f \equiv \partial_{x}^{-1}\left[\mathrm{e}^{i v} f\right], \partial_{\mathrm{e}^{-i v}}^{-1} f \equiv \partial_{x}^{-1}\left[\mathrm{e}^{-i v} f\right]
$$


for an arbitrary function $f$. Thus, the PMKdV hierarchy (positive hierarchy) and the sG hierarchy (negative hierarchy) can be written as

$$
v_{t_{2 n+1}}=\Phi^{n} v_{x}=\left(\partial_{x}^{2}+v_{x}^{2}-v_{x} \partial_{x}^{-1} v_{x x}\right)^{n} v_{x} \equiv K_{2 n+1}, n=0,1,2, \ldots
$$

and

$$
v_{\tau_{2 n+1}}=\Phi^{-n} \partial_{x}^{-1} \sin (v)=\left(\partial_{\sin }^{-2}+\partial_{\cos }^{-2}\right)^{n} \partial_{x}^{-1} \sin (v) \equiv K_{-2 n-1}, n=0,1,2, \ldots,
$$

respectively. It is known [62] that $K_{2 n+1}$ defined in (2.6) are local symmetries and $K_{-2 n-1}$ defined in (2.7) are nonlocal symmetries of the PMKdV equation (2.2) for all $n=0,1,2, \ldots$

First few flow equations of (2.6) and (2.7) are listed as follows

$$
\begin{aligned}
v_{t_{1}}= & v_{x} \\
v_{\tau_{1}} \equiv & v_{\tau}=\partial_{x}^{-1} \sin (v) \\
v_{t_{3}} \equiv & v_{t}=v_{x x x}+\frac{1}{2} v_{x}^{3} \\
v_{\tau_{3}}= & \frac{1}{6} v_{\tau}^{3}+\partial_{\cos }^{-2} v_{\tau}, v_{\tau}=\partial_{x}^{-1} \sin (v) \\
v_{t_{5}}= & v_{x x x x x}+\frac{5}{2} v_{x}^{2} v_{x x x}+\frac{5}{2} v_{x} v_{x x}^{2}+\frac{3}{8} v_{x}^{5} \\
v_{\tau_{5}}= & \frac{1}{120} v_{\tau}^{5}+\frac{1}{6} \partial_{\cos }^{-2} v_{\tau}^{3}+\left(\partial_{\sin }^{-2}+\partial_{\cos }^{-2}\right) \partial_{\cos }^{-2} v_{\tau}, \\
v_{t_{7}}= & v_{x x x x x x x}+\frac{7}{2} v_{x}^{2} v_{x x x x x}+14 v_{x} v_{x x} v_{x x x x}+\frac{21}{2} v_{x} v_{x x x}^{2}+\frac{35}{8} v_{x}^{4} v_{x x x} \\
& +\frac{35}{4}\left(2 v_{x x x}+v_{x}^{3}\right) v_{x x}^{2}+\frac{5}{16} v_{x}^{7}, \\
v_{\tau_{7}}= & \frac{1}{5040} v_{\tau}^{7}+\frac{1}{120} \partial_{\cos }^{-2} v_{\tau}^{5}+\frac{1}{6}\left(\partial_{\sin }^{-2}+\partial_{\cos }^{-2}\right) \partial_{\cos }^{-2} v_{\tau}^{3}+\left(\partial_{\sin }^{-2}+\partial_{\cos }^{-2}\right)^{2} \partial_{\cos }^{-2} v_{\tau} .
\end{aligned}
$$

Using the definition of the commutation relation $[F(v), G(v)]$ as

$$
[F(v), G(v)]=F^{\prime} G-G^{\prime} F=\lim _{\epsilon=0} \frac{\mathrm{d}}{\mathrm{d} \epsilon}[F(v+\epsilon G)-G(v+\epsilon F)],
$$

it is not difficult to prove that all the local symmetries $\Phi^{n} v_{x}=K_{2 n+1}$ and the nonlocal symmetries $\Phi^{-n} \partial_{x}^{-1} \sin (v)=K_{-2 n-1}$ commute each other [26, 62],

$$
\left[K_{2 n+1}, K_{2 m+1}\right]=\left[K_{2 n+1}, K_{-2 m-1}\right]=\left[K_{-2 n-1}, K_{-2 m-1}\right]=0, n, m=0,1,2, \ldots
$$

which means everyone of $K_{2 n+1}, K_{-2 n-1}$ and their linear combinations are solutions of the symmetry equations of the PMKdV and the sG hierarchies. The symmetry equation of the PMKdV equation is defined as

$$
\sigma_{t}=\sigma_{x x x}+\frac{3}{2} v_{x}^{2} \sigma_{x}
$$

which is the linearized equation of (2.10) and is obtained by substituting $v \rightarrow v+\epsilon \sigma$ with the infinitesimal parameter $\epsilon$ into the PMKdV equation (2.10). 
The symmetry equation of the sG equation,

$$
\sigma_{x \tau}=\cos (v) \sigma
$$

is the linearized form of eq. (2.1).

Because the $\mathrm{sG}$ equation (2.1) is relativistically invariant ( $x, \tau$ exchange invariance) and $\Phi$ given by (2.3) is a proper recursion operator for it, we immediately have the proposition 1.

Proposition 1. The operator

$$
\Phi_{1}=\partial_{\tau}^{2}+v_{\tau}^{2}-v_{\tau} \partial_{\tau}^{-1} v_{\tau \tau}
$$

is a recursion operator for the $s G$ equation (2.1).

It is observed that $\Phi_{1}$ is nothing but the inverse of the recursion operator $\Phi$ as implied by the following proposition 2 .

Proposition 2. If $\sigma$ is a symmetry of the $s G$ equation (2.1), i.e., a solution of (2.17), then

$$
\Phi_{1} \Phi \sigma=\sigma=\Phi \Phi_{1} \sigma
$$

Proof. The second equality follows from the first one under the exchange $x \leftrightarrow \tau$, so it is enough to prove the first equation of (2.19). Indeed, we have

$$
\begin{aligned}
\Phi_{1} \Phi \sigma= & \left(\partial_{x}^{2} \partial_{\tau}^{2}+v_{x}^{2} \partial_{\tau}^{2}+v_{\tau}^{2} \partial_{x}^{2}+2 v_{x \tau}^{2}\right) \sigma+v_{x \tau \tau}\left(2 v_{x}-\partial_{x}^{-1} v_{x x}\right) \sigma \\
& +2 v_{x \tau}\left(2 v_{x} \partial_{\tau}-\partial_{x}^{-1} \partial_{\tau} v_{x x}\right) \sigma+v_{x} v_{\tau}^{2}\left(v_{x}-\partial_{x}^{-1} v_{x x}\right) \sigma \\
& +v_{\tau} \partial_{\tau}^{-1}\left(v_{x} v_{\tau \tau} \partial_{x}^{-1} v_{x x}-v_{\tau \tau} v_{x}^{2}-v_{\tau \tau} \partial_{x}^{2}\right) \sigma-v_{x} \partial_{x}^{-1} \partial_{\tau}^{2} v_{x x} \sigma .
\end{aligned}
$$

Eliminating $v_{x \tau}$ and $\sigma_{x \tau}$ via the sG equation (2.1) and its symmetry equation (2.17), (2.20) yields

$$
\begin{aligned}
\Phi_{1} \Phi \sigma= & {\left[1+v_{x} v_{\tau} \cos (v)+v_{x}^{2} v_{\tau}^{2}+v_{x} \sin (v) \partial_{\tau}\right] \sigma-v_{x} v_{\tau}^{2} \partial_{x}^{-1} v_{x x} \sigma } \\
& -v_{x} \partial_{\cos }^{-1}\left[\sin (v)+v_{x} \partial_{\tau}\right] \sigma-v_{\tau} \cos (v) \partial_{x}^{-1} v_{x x} \sigma \\
& +v_{\tau}\left\{\partial_{\tau}^{-1}\left[v_{x} v_{\tau \tau}\left(\partial_{x}^{-1} v_{x x}-v_{x}\right)-\sin (v)\left(\cos (v)+v_{x} v_{\tau}\right)\right]\right\} \sigma
\end{aligned}
$$

Performing some integrations by parts and using the relations (2.1) and (2.17), it is straightforward to find that (2.21) is just the first equation of (2.19), and the proposition 2 is proved.

Because $\Phi_{1}$ expressed in (2.18) is just the inverse of $\Phi$, the sG hierarchy (2.7) (the negative PMKdV hierarchy) can be reformulated as

$$
v_{\tau_{2 n+1}}=\left(\partial_{\tau}^{2}+v_{\tau}^{2}-v_{\tau} \partial_{\tau}^{-1} v_{\tau \tau}\right)^{n} v_{\tau}, n=0,1,2, \ldots,
$$

with $v_{\tau}=\partial_{x}^{-1} \sin (v)$.

Thus, we call the positive PMKdV hierarchy (2.6) and the negative PMKdV hierarchy (2.7) (sG hierarchy) are dual each other while the sG equation is termed as the duality 
relation of the hierarchies. In other words, the local symmetries $K_{2 n+1}$ and the nonlocal symmetries $K_{-2 n-1}$ are dual with the duality relation

$$
v_{x} \leftrightarrow v_{\tau}\left[=\partial_{x}^{-1} \sin (v)\right]
$$

Alternatively, we can also say that the set of the nonlocal symmetries of the PMKdV equation can be localized with help of the duality relation (2.23). In fact, for the $n$th equation of the negative hierarchy (2.7) or (2.22) is local in $\left\{\tau, \tau_{2 n+1}\right\}$ space-time ( $\tau$ space and $\tau_{2 n+1}$ time) and nonlocal in $\left\{x, \tau_{2 n+1}\right\}$ space-time ( $x$ space and $\tau_{2 n+1}$ time). For the $n$th equation of the positive hierarchy (2.6) is local in $\left\{x, t_{2 n+1}\right\}$ space and nonlocal in $\left\{\tau, t_{2 n+1}\right\}$ space while $x$ and $\tau$ are related by the sG equation.

Summarizing above results, we have the following conjecture.

Dual conjecture of positive and negative hierarchies. For an integrable system, there exists a possible dual relation such that a positive hierarchy can be changed to a negative hierarchy.

If the positive hierarchy (like the PMKdV hierarchy) is local and the negative hierarchy is nonlocal (like the sG hierarchy), then the duality conjecture indicates that the local symmetries and nonlocal symmetries may be dual each other via a possible dual relation.

\section{Duality of the PFG hierarchy and the Tzitzeica hierarchy}

To provide further support for our conjecture, we consider another well known integrable system, the PFG equation [44],

$$
v_{t}=v_{x x x x x}-\frac{5}{2} v_{x x} v_{x x x}-\frac{5}{4} v_{x} v_{x x}^{2}-\frac{5}{4} v_{x}^{2} v_{x x x}+\frac{1}{16} v_{x}^{5} \equiv K_{5} .
$$

It is a potential form of the FG equation $\left(u=v_{x}\right)[44]$

$$
u_{t}=\left(u_{x x x x}-\frac{5}{2} u_{x} u_{x x}-\frac{5}{4} u u_{x}^{2}-\frac{5}{4} u^{2} u_{x x}+\frac{1}{16} u^{5}\right)_{x},
$$

which is a common modified system of the SK and KK equations via different Miura transformations. The SK and KK equations are two important physical models which can be used to describe all the physical fields where the KdV equation is not adequate and needs some higher order corrections [64].

From the results of the SK and KK equations, we know that the PFG equation possesses two sets of local symmetries, or equivalently, two positive hierarchies [63]

$$
v_{t_{6 n+1}}=\Psi_{D}^{n} v_{x} \equiv \Psi_{D}^{n} K_{1}, n=0,1,2, \ldots
$$

and

$$
v_{t_{6 n+5}}=\Psi_{D}^{n} K_{5}, n=0,1,2, \ldots
$$


with $K_{5}$ being defined in (3.1), the recursion operator and the inverse recursion operator being given by

$$
\begin{aligned}
\Psi_{D} & =D_{g} g^{-1} D_{g^{3}}^{-1} D_{g}^{5} g^{-2} D_{g^{2}}^{-1} D_{g}^{2}, g \equiv \exp \left(\frac{v}{2}\right) \\
\Psi_{D}^{-1} & =D_{g}^{-2} D_{g^{2}} g^{2} D_{g}^{-5} D_{g^{3}} g D_{g}^{-1} \\
& =\frac{1}{9}\left(\partial_{2}^{-1} \partial_{1}^{-1} \partial_{2}^{-2} \partial_{1}^{-1} \partial_{2}^{-1}+2 \partial_{1}^{-1} \partial_{2}^{-3} \partial_{1}^{-1} \partial_{2}^{-1}+2 \partial_{2}^{-1} \partial_{1}^{-1} \partial_{2}^{-3} \partial_{1}^{-1}+4 \partial_{1}^{-1} \partial_{2}^{-4} \partial_{1}^{-1}\right)
\end{aligned}
$$

where

$$
D_{f} \equiv f \partial_{x}, D_{f}^{-1} \equiv\left(D_{f}\right)^{-1}=\partial_{x}^{-1} f^{-1}, \partial_{1}^{-1} \equiv \partial_{x}^{-1} \mathrm{e}^{v}, \partial_{2}^{-1} \equiv \partial_{x}^{-1} \mathrm{e}^{-\frac{v}{2}}
$$

It is mentioned that the seed symmetries $K_{1}$ and $K_{5}$ are only two nontrivial solutions of $\Psi_{D}^{-1} f=0$.

The PFG equation is known to possess a nonlocal symmetry $\sigma=\partial_{x}^{-1}\left(\mathrm{e}^{v}+\mathrm{e}^{-\frac{v}{2}}\right)$ and the related flow is the well known Tzitzeica equation (TE)

$$
v_{x \tau}=\mathrm{e}^{v}+\mathrm{e}^{-\frac{v}{2}}
$$

It is obviously that the TE (3.7) is relativistically invariant (the space-time $(x-\tau)$ exchange invariance under the light cone coordinate). Thus, similar to the sG equation, all symmetries of the Tzitzeica equation continue to be symmetries under the $x \leftrightarrow \tau$ exchange transformation.

Based on the relativistic invariance of TE, we formulate the following proposition.

Proposition 3. If $\sigma$ is a symmetry of TE, i.e., a solution of

$$
\sigma_{x \tau}=\sigma \mathrm{e}^{v}-\frac{\sigma}{2} \mathrm{e}^{-\frac{v}{2}}
$$

then

$$
\Psi_{D} \Psi_{\Delta} \sigma=\sigma=\Psi_{\Delta} \Psi_{D} \sigma
$$

where

$$
\Psi_{\Delta}=\Delta_{g} g^{-1} \Delta_{g^{3}}^{-1} \Delta_{g}^{5} g^{-2} \Delta_{g^{2}}^{-1} \Delta_{g}^{2}, \Delta_{f} \equiv f \partial_{\tau}, \Delta_{f}^{-1} \equiv\left(\Delta_{f}\right)^{-1}=\partial_{\tau}^{-1} f^{-1}
$$

A similar argument as we made for proving the proposition 2 may be employed to prove above proposition, namely taking the Tzitzeica equation (3.7) and the linearized symmetry equation (3.9) into consideration and integrations by parts. The detailed calculations, which are rather cumbersome, may be implemented with the assistant of the computer algebras such MAPLE or MATHEMATICA. Thus, we omit the proof.

Thanks to the proposition 3, the dual hierarchy of the first positive hierarchy (3.2) can be written as

$$
v_{\tau_{6 n+1}}=\Psi_{\Delta}^{n} v_{\tau}, n=0,1,2, \ldots
$$


The first two of (3.11) read as

$$
\begin{aligned}
v_{\tau_{1}}= & v_{\tau}=\partial_{x}^{-1}\left(\mathrm{e}^{v}+\mathrm{e}^{-\frac{v}{2}}\right) \\
v_{\tau_{7}}= & v_{\tau \tau \tau \tau \tau \tau \tau}-\frac{7}{4}\left(v_{\tau}^{2}+2 v_{\tau \tau}\right) v_{\tau \tau \tau \tau \tau}-7\left(v_{\tau} v_{\tau \tau}+v_{\tau \tau \tau}\right) v_{\tau \tau \tau \tau} \\
& -\frac{7}{8} v_{\tau \tau \tau}\left(6 v_{\tau} v_{\tau \tau \tau}-2 v_{\tau}^{2} v_{\tau \tau}-v_{\tau}^{4}+8 v_{\tau \tau}^{2}\right)+\frac{7}{4} v_{\tau}^{3} v_{\tau \tau}^{2}+\frac{7}{6} v_{\tau} v_{\tau \tau}^{3}-\frac{1}{48} v_{\tau}^{7} \\
= & \Psi_{D}^{-1} \partial_{x}^{-1}\left(\mathrm{e}^{v}+\mathrm{e}^{-\frac{v}{2}}\right)=\Psi_{\Delta} v_{\tau} .
\end{aligned}
$$

For the second positive hierarchy (3.3), its dual hierarchy is

$$
v_{\tau_{6 n+5}}=\Psi_{\Delta}^{n}\left(v_{\tau \tau \tau \tau \tau}-\frac{5}{2} v_{\tau \tau} v_{\tau \tau \tau}-\frac{5}{4} v_{\tau} v_{\tau \tau}^{2}-\frac{5}{4} v_{\tau}^{2} v_{\tau \tau \tau}+\frac{1}{16} v_{\tau}^{5}\right), n=0,1,2, \ldots,
$$

whose simplest model of the hierarchy is the fifth order negative PFG equation

$$
\begin{aligned}
v_{\tau_{5}} & =v_{\tau \tau \tau \tau \tau}-\frac{5}{2} v_{\tau \tau} v_{\tau \tau \tau}-\frac{5}{4} v_{\tau} v_{\tau \tau}^{2}-\frac{5}{4} v_{\tau}^{2} v_{\tau \tau \tau}+\frac{1}{16} v_{\tau}^{5}=K_{5}^{-} \\
& =\partial_{1}^{-1} z^{4}+2 \partial_{2}^{-1} \partial_{1}^{-1} z^{3}+24 \partial_{1}^{-1} \partial_{2}^{-3} w+12 \partial_{2}^{-1} \partial_{1}^{-1} \partial_{2}^{-2} w
\end{aligned}
$$

with

$$
z_{x}=\mathrm{e}^{-\frac{v}{2}}, w_{x}=\mathrm{e}^{v}
$$

\section{Duality of the dKPL hierarchy and the two-dimensional dispersionless Toda hierarchy}

In (2+1)-dimensional cases, it is still possible to construct a positive integrable hierarchy with help of the FSSA [34] for certain nonlinear systems in the form

$$
u_{t x_{1}}=K\left(t, x_{1}, \ldots, x_{m}, u, u_{x_{1}}, u_{x_{2}}, \ldots\right) \equiv K(u), m \geq 2,
$$

where $K(u)$ is a function of the space time $\left(\left\{t, x_{1}, \ldots, x_{m}\right\}\right)$ and space $\left(\left\{x_{1}, \ldots, x_{m}\right\}\right)$ derivations of $u$ but not dependent on time $(t)$ derivatives of $u$. The (2+1)-dimensional nonlinear system (4.1) possesses a formal series symmetry thanks to the following proposition.

Proposition 4. [34] Let $f=f(t)$ and $g=g\left(t, x_{2}, \ldots, x_{m}\right)$ be arbitrary functions of the indicated variables, then

$$
\sigma(f, g)=\sum_{k=0}^{\infty} f^{(-k)}\left(\partial_{x}^{-1} K^{\prime}-\partial_{t}\right)^{k} g
$$

is a formal symmetry of (4.1) with $f^{(-k)} \equiv \partial_{t}^{-k} f$ and

$$
K^{\prime} h \equiv \lim _{\epsilon \rightarrow 0} \frac{\mathrm{d}}{\mathrm{d} \epsilon} K(u+\epsilon h) .
$$


While it is not possible to prove the convergence for the formal series symmetry (4.2) with arbitrary $f$ and $g$ in general, it is fortunate and interesting that for various $(2+1)$ dimensional integrable systems such as the KP equation [65], the Toda field [34], the Nizhnik-Novikov-Veselov equation [66], the dispersive long wave equation [67] and the differential-difference Toda equation [68], the formal series symmetries can be truncated to a closed summation form by selecting the arbitrary function $g$ as special polynomial functions.

Whence the formal series symmetry (4.2) is truncated up to the $n$th term by fixing $g=g_{n}$, we can rewrite (4.2) as

$$
\sigma_{n}(f)=\sum_{k=0}^{n} f^{(n-k)}\left(\partial_{x}^{-1} K^{\prime}-\partial_{t}\right)^{k} g_{n}
$$

after changing $f$ to $f^{(n)}$ because of its arbitrariness. More specifically, by fixing the arbitrary function $f$ as a constant, say, $f=1$, one may obtain an integrable (positive) hierarchy

$$
u_{t_{n}}=\left(\partial_{x}^{-1} K^{\prime}-\partial_{t}\right)^{n} g_{n}
$$

if (4.1) is integrable.

Now we consider the following equation

$$
v_{y t}=\left(v v_{x}\right)_{y}-v_{x x},
$$

which was proposed recently by Zakharov et al. [69]. This (2+1)-dimensional equation, referred as the dispersionless Kadomtsev-Petviashvili-like (dKPL) equation, resembles the dispersionless Kadomtsev-Petviashvili (dKP) equation

$$
v_{x t}=\left(v v_{x}\right)_{x}-v_{y y},
$$

and the dispersionless negative BKP equation

$$
P_{y t}=3\left(P_{x} P_{y}\right)_{x}-3 P_{x x} .
$$

It is noted that (4.5), (4.6) and (4.7) are different (2+1)-dimensional extensions of the Riemann equation $w_{t}=w w_{x}$.

The potential form of the dKPL equation (4.5) reads as

$$
u_{y t}=\frac{1}{2} u_{x y}^{2}-u_{x x} \equiv G
$$

where $v=u_{x y}$. Applying the proposition 4 to it and fixing the arbitrary function $g$ as $g=-\frac{1}{4 n !} x^{n}$, we find an integrable positive potential dKPL hierarchy in the form

$$
u_{t_{n-2}}=-\frac{1}{4 n !}\left(\partial_{y}^{-1} u_{x y} \partial_{x} \partial_{y}-\partial_{y}^{-1} \partial_{x}^{2}-\partial_{t}\right)^{n-1} x^{n}, n=3,4, \cdots .
$$

The first four flows of this hierarchy (4.9) (for $n=3,4,5$ and 6 , respectively) read

$$
\begin{aligned}
u_{t_{1}} & =\frac{1}{4} u_{x}, \\
u_{y t_{2}} & =\frac{1}{2} u_{x y}^{2}-u_{x x},
\end{aligned}
$$


which is just the potential dKPL equation (4.8) with $t_{2}=t$,

$$
u_{y t_{3}}=\frac{1}{6} u_{x y}^{3}-\frac{1}{2} u_{x x} u_{x y}-\frac{1}{2} v, v_{y}=G_{x},
$$

with $G$ being defined in (4.8) and

$$
u_{y y t_{4}}=\frac{3}{4}\left(u_{x y y}-\partial_{x}\right)\left(v+2 u_{x x} u_{x y}-u_{x y}^{3}\right) .
$$

In general, recursion operators are not available for $(2+1)$-dimensional integrable systems, so we have to adopt other methods $[30,35]$ to find the related negative hierarchy. For a given $(2+1)$-dimensional system, provided that a symmetry flow,

$$
u_{x \tau}=F(u)
$$

which possesses the space-time $\{x, \tau\}$ exchange invariance, then we may construct a dual negative hierarchy by using the duality relation $u_{\tau}=\partial_{x}^{-1} F(u)$. Fortunately, for the potential dKPL equation (4.8) we have the following proposition.

Proposition 5. The equation

$$
u_{\tau}=\partial_{x}^{-1} \mathrm{e}^{-u_{y y}}
$$

constitutes a symmetry of the potential dKPL equation (4.8).

Proof. To prove it, we need to show $\sigma=\partial_{x}^{-1} \mathrm{e}^{-u_{y y}}$ solves the linearized potential dKPL equation, namely

$$
\sigma_{y t}-u_{x y} \sigma_{x y}+\sigma_{x x}=0
$$

Substituting $\sigma=\partial_{x}^{-1} \mathrm{e}^{-u_{y y}}$ into the left-hand side of (4.12), we have

$$
\left(u_{x y} u_{y y y}-u_{x y y}\right) \mathrm{e}^{-u_{y y}}+\partial_{x}^{-1}\left[\left(u_{y y y} u_{y y t}-u_{y y y t}\right) \mathrm{e}^{-u_{y y}}\right] .
$$

After using the potential dKPL equation, (4.13) becomes

$$
\begin{aligned}
& \left(u_{x y} u_{y y y}-u_{x y y}\right) \mathrm{e}^{-u_{y y}}+\partial_{x}^{-1}\left[\mathrm{e}^{-u_{y y}}\left(u_{y y y} \partial_{y}-\partial_{y}^{2}\right) G\right] \\
& \quad=\partial_{x}^{-1}\left\{\partial_{x}\left[\left(u_{x y} u_{y y y}-u_{x y y}\right) \mathrm{e}^{-u_{y y}}\right]+\mathrm{e}^{-u_{y y}}\left(u_{y y y} \partial_{y}-\partial_{y}^{2}\right) G\right\} \\
& =\partial_{x}^{-1}\left[\left(u_{y y y}-\partial_{y}\right)\left(G_{y}+u_{x x y}-u_{x y} u_{x y y}\right) \mathrm{e}^{-u_{y y}}\right],
\end{aligned}
$$

which vanishes due to the definition of $G$. Thus the proposition 5 is proved.

The equation (4.11) or

$$
u_{x \tau}=\mathrm{e}^{-u_{y y}}
$$

is the large $N$ limit $(N \rightarrow \infty)$ of the two-dimensional $\operatorname{sl}(N+1)$ Toda field [70-73] which is also known as the Boyer-Finley equation [74] or the $\mathrm{SU}(\infty)$ Toda equation [75] or the twodimensional dispersionless Toda (2ddT) equation [76]. Because of the relativistic invariance of the $2 \mathrm{ddT}$ equation (4.14), we can build a hierarchy (negative dKPL hierarchy) which is a dual hierarchy of the positive potential dKPL hierarchy (4.9)

$$
u_{\tau_{n-2}}=-\frac{1}{4 n !}\left(\partial_{y}^{-1} u_{y \tau} \partial_{\tau} \partial_{y}-\partial_{y}^{-1} \partial_{\tau}^{2}-\partial_{t}\right)^{n-1} \tau^{n}, n=3,4, \cdots,
$$

with $\tau$ being defined by the duality relation (4.11). 
The first three equations of the hierarchy (4.15) possess the following forms

$$
u_{x \tau_{1}}=\frac{1}{4} \mathrm{e}^{-u_{y y}}=\frac{1}{4} u_{x \tau},
$$

which is just the $2 \mathrm{ddT}$ equation (4.14) with $\tau_{1}=4 \tau$,

$$
u_{x y \tau_{2}}=\left(z u_{y y y}-z_{y}\right) \mathrm{e}^{-u_{y y}}, z_{x}=u_{y y y} \mathrm{e}^{-u_{y y}},
$$

and

$$
u_{x y y \tau_{3}}=z_{x} w_{y}+\frac{1}{2} w z_{x y}-\frac{1}{2} w_{y y} \mathrm{e}^{-u_{y y}}, w_{x}=\left(z_{y}-2 z u_{y y y}\right) \mathrm{e}^{-u_{y y}} .
$$

\section{Duality hierarchies from the two-dimensional Toda lattice}

It is known that the $2 \mathrm{ddT}$ equation (4.14) is an integrable continuous limit of the following differential-difference system [48, 68, 77, 78]

$$
u_{x \tau}=\mathrm{e}^{u_{n-1}-u_{n}}-\mathrm{e}^{u_{n}-u_{n+1}} \equiv A-A_{1}=-\Delta A_{1}, A_{k} \equiv \mathrm{e}^{-\Delta u_{n+k}}, \Delta f_{n}=f_{n}-f_{n-1},
$$

which is the celebrated two-dimensional Toda lattice (2dTL). In this section, we aim to construct possible dual systems by taking the 2dTL (5.1), which is relativistically invariant, as a duality relation. To this end, we should first build a hierarchy (negative hierarchy) related to (5.1) and this will be done by means of the mastersymmetry method [33].

A direct calculation yields

$$
[n, K]=K_{1}=K=\partial_{x}^{-1}\left[\mathrm{e}^{u_{n-1}-u_{n}}-\mathrm{e}^{u_{n}-u_{n+1}}\right]=u_{\tau}=u_{\tau_{1}},
$$

where the commutator is defined as

$$
[F, G]=F^{\prime} G-G^{\prime} F=\lim _{\epsilon=0} \frac{\mathrm{d}}{\mathrm{d} \epsilon}\left[F\left(u_{n}+\epsilon G\right)-G\left(u_{n}+\epsilon F\right)\right] .
$$

Therefore, while $n$ is not a symmetry of the $2 \mathrm{dTL}(5.1)$, it is actually a mastersymmetry. To find the next (higher order) flow one can apply the higher order mastersymmetry $n^{2}$. Some simple calculations result in

$$
\left[\left[n^{2}, K\right], K\right]=2 \Delta \partial_{x}^{-1} A_{1} \partial_{x}^{-1}\left(A_{2}-A\right) \equiv K_{2}=u_{\tau_{2}},
$$

which, after taking the 2dTL (5.1) into consideration, may be rewritten as

$$
u_{x \tau_{2}}=2\left(u_{n}+u_{n-1}\right)_{\tau} \mathrm{e}^{u_{n-1}-u_{n}}-2\left(u_{n}+u_{n+1}\right)_{\tau} \mathrm{e}^{u_{n}-u_{n+1}}=-4 \Delta A_{1} E u_{n+1, \tau},
$$

where the average operator $E$ is defined by

$$
E f_{n}=\frac{1}{2}\left(f_{n}+f_{n-1}\right)
$$

and $\tau$ is related to $x$ by the $2 \mathrm{dTL}$ (5.1). Under $x=\tau,(5.4)$ reduces to an equation appeared early $[79,80]$. 
Now taking (5.1) as a duality relation, we may work out the dual equation (the equation of the related positive hierarchy) of (5.4) in the $\left\{\tau, \tau_{2}\right\}$ space. Indeed, eliminating $u_{x}$ by means of (5.1), (5.4) becomes

$$
\Delta\left\{A_{1}\left[\Delta\left(u_{n+1, \tau_{2}}-2 u_{n+1, \tau}^{2}\right)+4 E u_{n+1, \tau \tau}\right]\right\}=0,
$$

which leads to

$$
\Delta u_{n \tau_{2}}=2 \Delta u_{n \tau}^{2}-4 E u_{n \tau \tau}
$$

or

$$
u_{n \tau_{2}}=2 u_{n \tau}^{2}-4 \Delta^{-1} E u_{n \tau \tau}, \Delta^{-1} \Delta=1
$$

Applying the relativistic invariance again, we have another but equivalent integrable model

$$
u_{n \tau_{2}}=2 u_{n x}^{2}-4 \Delta^{-1} E u_{n x x} \equiv K_{2}^{+} .
$$

It is interesting to note that the new equation (5.7) just constructed may be taken as a semi-discrete potential dKPL equation (4.8) where the variable $y$ is discretized.

Directly we may check that

$$
u_{n \tau_{2} \tau}-u_{n \tau \tau_{2}}=\left[K_{2}^{+}, K\right]=0
$$

holds, which also implies $\left[\left[\left[n^{2}, K\right], K\right], K\right]=0$. In other words, both $K_{2}$ and $K_{2}^{+}$defined in (5.3) and (5.7) are symmetries and $n^{2}$ is a mastersymmetry of the 2dTL (5.1).

\section{From the real second heavenly equation to dual hierarchies}

In this section, we apply the similar duality approach to find positive and negative heavenly hierarchies by means of the exchange invariance

$$
\{x, \tau, y, z, u\} \longleftrightarrow\{\tau, x, z, y,-u\}
$$

of the real second heavenly equation [81]

$$
u_{x z}-u_{y \tau}+u_{z z} u_{y y}-u_{y z}^{2}=0 .
$$

The heavenly equations, introduced in [81] by Plebanski, describe self-dual vacuum solutions of the Einstein equations. The equation (6.2) has been studied extensively and many results have been established (see [82-85] and the references therein). In particular, the multi-Hamiltonian structures and assocaited (positive) hierarchies have been explored by different methods [85-87].

According to the FSSA and the mastersymmetry method as discussed in the sections 4 and 5 , we can find that the heavenly equation possesses two positive hierarchies in the forms

$$
u_{t_{n-1}}=\left[\partial_{y}^{-1}\left(\partial_{x} \partial_{z}+u_{z z} \partial_{y}^{2}+u_{y y} \partial_{z}^{2}-2 u_{y z} \partial_{y} \partial_{z}\right)-\partial_{\tau}\right]^{n-1} \frac{z^{n}}{n !}, n=1,2, \ldots
$$

and

$$
u_{\tau_{n-1}}=\left[\partial_{z}^{-1}\left(\partial_{\tau} \partial_{y}-u_{z z} \partial_{y}^{2}-u_{y y} \partial_{z}^{2}+2 u_{y z} \partial_{y} \partial_{z}\right)-\partial_{x}\right]^{n-1} \frac{y^{n}}{n !}, n=1,2, \ldots
$$


It is clear that above two hierarchies are related via the discrete symmetry transformation (6.1).

In the positive hierarchy (6.3) the variable $\tau$ should be eliminated by means of the heavenly equation (6.2), i.e.,

$$
u_{\tau}=\partial_{y}^{-1}\left(u_{x z}+u_{z z} u_{y y}-u_{y z}^{2}\right) \equiv K .
$$

For the second positive hierarchy (6.4), we should eliminate the variable $x$ via

$$
u_{x}=\partial_{z}^{-1}\left(u_{y \tau}-u_{z z} u_{y y}+u_{y z}^{2}\right) \equiv P .
$$

After some tedious calculations, we find that the hierarchy (6.3) may be reformulated concisely as

$$
u_{t_{n}}=\Phi^{n} z=\Phi^{n-2} u_{x}, \Phi \equiv \partial_{y}^{-1}\left(u_{y y} \partial_{z}-u_{y z} \partial_{y}+\partial_{x}\right)
$$

which for $n=1,2,3$ leads to

$$
\begin{aligned}
u_{t_{1}} & =u_{y}=\Phi z \\
u_{t_{2}} & =u_{x}=\Phi^{2} z \\
u_{y t_{3}} & =u_{y y} u_{x z}-u_{y z} u_{x y}+u_{x x}=\left(\Phi^{3} z\right)_{y},
\end{aligned}
$$

while the second hierarchy (6.4) can be written as

$$
u_{\tau_{n}}=\Psi^{n} y=\Psi^{n-2} u_{z}, \Psi \equiv \partial_{z}^{-1}\left(\partial_{\tau}-u_{z z} \partial_{y}+u_{y z} \partial_{z}\right)
$$

with the first three examples

$$
\begin{aligned}
u_{\tau_{1}} & =-u_{z}=\Psi y, \\
u_{\tau_{2}} & =-u_{\tau}=\Psi^{2} y, \\
u_{z \tau_{3}} & =u_{y y} u_{z \tau}-u_{y z} u_{y \tau}-u_{\tau \tau}=\left(\Psi^{3} y\right)_{z} .
\end{aligned}
$$

The dual negative hierarchy of (6.7) can be obtained by using the relativistic invariance (6.1). The result reads as

$$
u_{\tau_{n}}=\left.\Psi^{n-2} u_{z}\right|_{u_{\tau}=K},
$$

and explicitly its first nontrivial flow (for $n=3$ ) is

$$
\begin{aligned}
u_{y \tau_{3}} & =\left.\partial_{z}^{-1}\left(u_{y \tau} u_{z z}-u_{y z} u_{z \tau}-u_{\tau \tau}\right)_{y}\right|_{u_{\tau}=K} \\
& =v_{z} u_{y y}-v_{y} u_{z y}+v_{x} \\
v_{y} & =u_{y z}^{2}-u_{y y} u_{z z}-u_{z x}
\end{aligned}
$$

In the same way, the dual negative hierarchy of (6.9) has the form

$$
u_{t_{n}}=\left.\Phi^{n-2} u_{x}\right|_{u_{x}=P} .
$$

Above discussions indicate that both $\Phi$ and $\Psi$ should be the recursion operators of the second heavenly equation (6.2). Indeed, we have 
Proposition 6. Let $\sigma$ be a symmetry of the second heavenly equation (6.2), i.e., a solution of

$$
\sigma_{y \tau}-\sigma_{x z}-\sigma_{z z} u_{y y}-u_{z z} \sigma_{y y}+2 u_{y z} \sigma_{y z}=0
$$

so are $\Phi \sigma$ and $\Psi \sigma$.

Proof. Making the change $\sigma \rightarrow \Phi \sigma$ and eliminating $\sigma_{\tau}$ and $u_{\tau}$ by means of (6.12) and (6.2), we have

$$
\begin{aligned}
\partial_{y}\left(\partial_{\tau}\right. & \left.-K^{\prime}\right)\left.\Phi \sigma\right|_{(6.2)(6.12)} \\
& =u_{y y}\left\{\left(u_{y z} \sigma_{z}-u_{z z} \sigma_{y}\right)_{z}+\partial_{y}^{-1}\left[\left(u_{z z} \sigma_{y y}-u_{y y z} \sigma_{z}\right)_{z}-u_{y z} \sigma_{y z z}+u_{y z z z} \sigma_{y}\right]\right\} \\
& =u_{y y} \partial_{y}^{-1}\left[\partial_{y}\left(u_{y z} \sigma_{z}-u_{z z} \sigma_{y}\right)_{z}+\left(u_{z z} \sigma_{y y}-u_{y y z} \sigma_{z}\right)_{z}-u_{y z} \sigma_{y z z}+u_{y z z z} \sigma_{y}\right] \\
& =u_{y y} \partial_{y}^{-1} 0=0 .
\end{aligned}
$$

So $\Phi \sigma$ is also a symmetry of (6.2). The conclusion for $\Psi \sigma$ also holds due to (6.1). Thus the proposition is proved.

Several remarks are in order:

Remark 1. Though the hierarchy (6.10) is a dual hierarchy of (6.7), we should mention that $\Psi$ is not a inverse of $\Phi$.

Remark 2. While we constructed the operators $\Phi$ and $\Psi$ via the FSSA, they did appear in a early work by Dunajski and Mason $[85,86]$.

Remak 3. Two recursion operators $\Phi$ and $\Psi$ commute each other, i.e.,

$$
[\Phi, \Psi] \sigma=(\Phi \Psi-\Psi \Phi) \sigma=0
$$

where $\sigma$ is a symmetry of the second heavenly equation (6.2).

According to the remark 3, two hierarchies (6.7) and (6.9) can be uniformly written as

$$
u_{t_{n m}}=\left.\Phi^{n} \Psi^{m} \sigma_{0,0}\right|_{(6.2)}, \sigma_{0,0}=x \text { or } \tau,
$$

while the dual hierarchy of (6.14) reads

$$
u_{t_{m n}}=\left.\Phi^{m} \Psi^{n} \sigma_{0,0}\right|_{(6.2)}, \sigma_{0,0}=\tau \text { or } x .
$$

\section{$7 \quad$ Summary and discussions}

In summary, if there is a relativistically invariant flow for an integrable nonlinear system, then the related positive and negative hierarchies are dual each other simply by taking the relativistically invariant flow as the duality relation. In (1+1)-dimensional cases, the positive hierarchies are local in a proper space $\left(\left\{x, t_{n}\right\}\right)$ and nonlocal in its dual space $\left(\left\{\tau, t_{n}\right\}\right)$ where the negative hierarchies are local in the space $\left\{\tau, \tau_{n}\right\}$ and nonlocal in the dual space $\left\{x, \tau_{n}\right\}$. Because of the existence of the recursion operators for $(1+1)$ dimensional integrable systems, we find that the recursion operators and their inverses and 
then the positive hierarchies and negative hierarchies possess completely same forms but with different "space" variables which are linked each other by means of the relativistic duality relations. This special structure will undoubtedly bring a lot of convenience when we deduce the integrable properties of the whole hierarchies under consideration. For example, by the dependent variable transformation, we can establish the following unified bilinear form for the whole potential $\mathrm{mKdV}$ hierarchy

$$
\begin{aligned}
& \left(D_{2 n+1}-D_{x}^{2} D_{2 n-1}\right) f^{*} \cdot f=0, \\
& \left(D_{-(2 n+1)}-D_{\tau}^{2} D_{-(2 n-1)}\right) f^{*} \cdot f=0, \\
& D_{x}^{2} f^{*} \cdot f=0, \quad D_{\tau}^{2} f^{*} \cdot f=0 \\
& D_{x} D_{\tau} f \cdot f=\frac{1}{2}\left(f^{2}-f^{* 2}\right)
\end{aligned}
$$

where $D_{2 k+1} \equiv D_{t_{2 k+1}}$ and $D_{-(2 k+1)} \equiv D_{\tau_{2 k+1}}$ are Hirota's bilinear operators defined by

$$
\begin{gathered}
D_{t}^{m} D_{x}^{n} a(t, x) \cdot b(t, x)=\left.\frac{\partial^{m}}{\partial s^{m}} \frac{\partial^{n}}{\partial y^{n}} a(t+s, x+y) b(t-s, x-y)\right|_{s=0, y=0}, \\
m, n=0,1,2, \cdots .
\end{gathered}
$$

Furthermore, starting from unified bilinear form for the whole potential mKdV hierarchy, we can derive the Bäcklund transformation and nonlinear superposition formula. Besides, it might be of interest to study the following equations of $x \leftrightarrow y$ invariance from geometric point of view,

$$
\begin{array}{rlrl}
v_{t}= & v_{x x x}+v_{y y y}+\frac{1}{2}\left(v_{x}^{3}+v_{y}^{3}\right), & v_{x y} & =\sin v, \\
v_{z t}= & \left(v_{x z}^{2}+v_{y z}^{2}\right)-2\left(v_{x x}+v_{y y}\right), & v_{x y} & =\mathrm{e}^{-v_{z z}}, \\
v_{n t}= & \left(v_{n y}^{2}+v_{n x}^{2}\right)-2 \Delta^{-1} E\left(v_{n y y}+v_{n x x}\right), & v_{n x y} & =\mathrm{e}^{v_{n-1}-v_{n}}-\mathrm{e}^{v_{n}-v_{n+1}}, \\
v_{t}= & v_{x x x x x}-\frac{5}{4}\left(2 v_{x x} v_{x x x}+v_{x} v_{x x}^{2}+v_{x}^{2} v_{x x x}\right)+\frac{1}{16} v_{x}^{5} \\
& +v_{y y y y y}-\frac{5}{4}\left(2 v_{y y} v_{y y y}+v_{y} v_{y y}^{2}+v_{y}^{2} v_{y y y}\right)+\frac{1}{16} v_{y}^{5}, v_{x y}=\mathrm{e}^{v}+\mathrm{e}^{-v / 2} .
\end{array}
$$

For a (2+1)-dimensional integrable model, the formal series symmetry approach [34] and the mastersymmetry method [33] can be readily used to find positive hierarchies. The negative hierarchies can be obtained by means of Lax operators [35] or the nonlocal symmetries like the squared eigenfunction symmetries and infinitesimal Bäcklund/Darboux transformations [30]. In this paper, the positive dKPL hierarchy is constructed within the framework of the FSSA while the negative hierarchy is found by the duality relation owing to the relativistic invariance of the two-dimensional dispersionless Toda equation. On the other hand, if one has a relativistically invariant integrable system, then it is possible to directly find the related dual hierarchies by using the system as duality relation. By combining the mastersymmetry method and the duality approach, the differential-difference dKPL equation (5.5) and the related higher order dual Toda equation are successfully obtained from the two-dimensional Toda lattice. In fact, there are many other relativistically 
invariant integrable systems such as the coupled Tzitzeica-sinh-Gordon model [88], the multicomponent sinh-Gordon systems [88], the Pohlmeyer-Lund-Regge-Getmanov model [8991], the principal SU(n) chiral model [92], the massive Thiring model [93], O(n) nonlinear $\sigma$ model [89], the self-dual Yang-Mills equation [94] and so on. For those relativistically invariant integrable systems, it is interesting to construct the related positive and negative dual hierarchies by adopting the duality method proposed in this paper.

In higher dimensions, the second heavenly equation (6.2) is especially important because it can be derived from both the Einstein's general relativistic equation and the selfdual Yang-Mills equation. Though the recursion operators of the second heavenly equation have been studied by Dunajski and Mason via the twistor theory of the anti-self-dual Einstein vacuum equations $[85,86]$, those operators are reobtained here directly by the simple FSSA. Using the heavenly equation as a duality relation the positive and negative second heavenly hierarchies are naturally obtained.

\section{Acknowledgments}

The work was sponsored by the National Natural Science Foundations of China (Nos. 11975131, 11871471 and 11931017) and K. C. Wong Magna Fund in Ningbo University.

Open Access. This article is distributed under the terms of the Creative Commons Attribution License (CC-BY 4.0), which permits any use, distribution and reproduction in any medium, provided the original author(s) and source are credited.

\section{References}

[1] P.J. Coles, M. Berta, M. Tomamichel and S. Wehner, Entropic uncertainty relations and their applications, Rev. Mod. Phys. 89 (2017) 015002.

[2] A. Slobozhanyuk, S.H. Mousavi, X. Ni, D. Smirnova, Y.S. Kivshar and A.B. Khanikaev, Three-dimensional all-dielectric photonic topological insulator, Nature Photon. 11 (2016) 130.

[3] X. Dong, D. Harlow and A.C. Wall, Reconstruction of Bulk Operators within the Entanglement Wedge in Gauge-Gravity Duality, Phys. Rev. Lett. 117 (2016) 021601 [arXiv: 1601.05416] [INSPIRE].

[4] N. Seiberg, T. Senthil, C. Wang and E. Witten, A Duality Web in 2+1 Dimensions and Condensed Matter Physics, Annals Phys. 374 (2016) 395 [arXiv:1606.01989] [InSPIRE].

[5] A. Karch and D. Tong, Particle-Vortex Duality from 3d Bosonization, Phys. Rev. X 6 (2016) 031043 [arXiv: 1606. 01893] [INSPIRE].

[6] X.N. Gao, S.Y. Lou and X.Y. Tang, Bosonization, singularity analysis, nonlocal symmetry reductions and exact solutions of supersymmetric KdV equation, JHEP 05 (2013) 029 [arXiv: 1308.6695] [INSPIRE].

[7] V.V. Bazhanov, S.L. Lukyanov and B.A. Runov, Bukhvostov-Lipatov model and quantum-classical duality, Nucl. Phys. B 927 (2018) 468 [arXiv:1711.09021] [INSPIRE].

[8] E.S. Abers and B.W. Lee, Gauge Theories, Phys. Rept. 9 (1973) 1 [InSPIRE]. 
[9] J.E. Kim, P. Langacker, M. Levine and H.H. Williams, A Theoretical and Experimental Review of the Weak Neutral Current: A Determination of Its Structure and Limits on Deviations from the Minimal SU(2) $-L \times \mathrm{U}(1)$ Electroweak Theory, Rev. Mod. Phys. 53 (1981) 211 [INSPIRE].

[10] S.Y. Lou and G.-J. Ni, Gaussian Effective Potential Method for $\mathrm{SU}(2) \times \mathrm{U}(1)$ Gauge Theory and Bounds on the Higgs Boson Mass, Phys. Rev. D 40 (1989) 3040 [inSPIRE].

[11] ATLAS collaboration, Observation of a new particle in the search for the Standard Model Higgs boson with the ATLAS detector at the LHC, Phys. Lett. B 716 (2012) 1 [arXiv: 1207.7214] [INSPIRE].

[12] CMS collaboration, Observation of a New Boson at a Mass of $125 \mathrm{GeV}$ with the CMS Experiment at the LHC, Phys. Lett. B $\mathbf{7 1 6}$ (2012) 30 [arXiv:1207.7235] [INSPIRE].

[13] P.A. Clarkson, Nonclassical symmetry reductions of the boussinesq equation, Chaos Solitons Fractals 5 (1995) 2261.

[14] Y.-Q. Li, J.-C. Chen, Y. Chen and S.Y. Lou, Darboux transformations via lie point symmetries: KdV equation, Chin. Phys. Lett. 31 (2014) 010201.

[15] S.-J. Liu, X.-Y. Tang and S.Y. Lou, Multiple Darboux-Bäcklund transformations via truncated Painlevé expansion and Lie point symmetry approach, Chin. Phys. B 27 (2018) 060201 [INSPIRE].

[16] P.A. Clarkson and M.D. Kruskal, New similarity reductions of the boussinesq equation, J. Math. Phys. 30 (1989) 2201.

[17] P.A. Clarkson and E.L. Mansfield, Algorithms for the nonclassical method of symmetry reductions, SIAM J. Appl. Math. 54 (1994) 1693.

[18] S.Y. Lou, Similarity solutions of the kadomtsev-petviashvili equation, J. Phys. A 23 (1990) L649.

[19] S.Y. Lou, H.-Y. Yuan, D.-F. Chen and W.-Z. Chen, Similarity reductions of the KP equation by a direct method, J. Phys. A 24 (1991) 1455 [InSPIRE].

[20] C.W. Cao and X.G. Geng, Neumann and Bargmann systems associated with the coupled KdV soliton hierarchy, J. Phys. A 23 (1991) 4117.

[21] Y. Cheng and Y. shen Li, The constraint of the kadomtsev-petviashvili equation and its special solutions, Phys. Lett. A 157 (1991) 22.

[22] B. Konopelchenko, J. Sidorenko and W. Strampp, (1+1)-dimensional integrable systems as symmetry constraints of (2+1)-dimensional systems, Phys. Lett. A 157 (1991) 17.

[23] S.Y. Lou and X.-B. Hu, Infinitely many lax pairs and symmetry constraints of the KP equation, J. Math. Phys. 38 (1997) 6401.

[24] S.Y. Lou and R.X. Yao, Primary branch solutions of first order autonomous scalar partial differential equations via lie symmetry approach, J. Nonlin. Math. Phys. 24 (2017) 379.

[25] P.J. Olver, Evolution Equations Possessing Infinitely Many Symmetries, J. Math. Phys. 18 (1977) 1212 [INSPIRE].

[26] S.Y. Lou, Integrable models constructed from the symmetries of the modified KdV equation, Phys. Lett. B 302 (1993) 261 [INSPIRE].

[27] H. Aratyn, E. Nissimov and S. Pacheva, Method of squared eigenfunction potentials in integrable hierarchies of KP type, Commun. Math. Phys. 193 (1998) 493. 
[28] S.Y. Lou and X.-B. Hu, Non-local symmetries via darboux transformations, J. Phys. A 30 (1997) L95.

[29] X.-P. Cheng, C.-L. Chen and S.Y. Lou, Interactions among different types of nonlinear waves described by the Kadomtsev-Petviashvili equation, Wave Motion 51 (2014) 1298.

[30] X.-B. Hu, S.Y. Lou and X.-M. Qian, Nonlocal symmetries for bilinear equations and their applications, Stud. Appl. Math. 122 (2009) 305.

[31] S.Y. Lou, X. Hu and Y. Chen, Nonlocal symmetries related to bäcklund transformation and their applications, J. Phys. A 45 (2012) 155209.

[32] S.Y. Lou, Conformal invariance and integrable models, J. Phys. A 30 (1997) 4803.

[33] B. Fuchssteiner, Mastersymmetries, higher order time-dependent symmetries and conserved densities of nonlinear evolution equations, Prog. Theor. Phys. 70 (1983) 1508.

[34] S.Y. Lou, Generalized symmetries and W(infinity) algebras in three-dimensional Toda field theory, Phys. Rev. Lett. 71 (1993) 4099 [inSPIRE].

[35] S.Y. Lou, Negative Kadomtsev-Petviashvili hierarchy, Phys. Scripta 57 (1998) 481.

[36] D.J. Korteweg and G. de Vries, XLI. on the change of form of long waves advancing in a rectangular canal, and on a new type of long stationary waves, Philos. Mag. 39 (1895) 422.

[37] D.G. Crighton, Applications of KdV, Acta Appl. Math. 39 (1995) 39.

[38] C.S. Gardner, J.M. Greene, M.D. Kruskal and R.M. Miura, Method for solving the Korteweg-deVries equation, Phys. Rev. Lett. 19 (1967) 1095 [INSPIRE].

[39] P. Minnhagen, The two-dimensional Coulomb gas, vortex unbinding, and superfluid-superconducting films, Rev. Mod. Phys. 59 (1987) 1001 [INSPIRE].

[40] K. Sawada and T. Kotera, A method for finding $N$-soliton solutions of the KdV equation and KdV-like equation, Prog. Theor. Phys. 51 (1974) 1355.

[41] P.J. Caudrey, R.K. Dodd and J.D. Gibbon, A new hierarchy of Korteweg-de Vries equations, Proc. Roy. Soc. Lond. A 351 (1976) 407.

[42] D.J. Kaup, On the inverse scattering problem for cubic eigenvalue problems of the class $\psi_{x x x}+6 Q \psi_{x}+6 R \psi=\lambda \psi$, Stud. Appl. Math. 62 (1980) 189.

[43] B.A. Kupershmidt, A Super Korteweg-De Vries Equation: An Integrable System, Phys. Lett. A 102 (1984) 213 [INSPIRE].

[44] A.P. Fordy and J. Gibbons, Some remarkable nonlinear transformations, Phys. Lett. A $\mathbf{7 5}$ (1980) 325.

[45] G. Tzitzéica, Sur une nouvelle classes de surfaces, C.R. Acad. Sci. Paris 144 (1907) 1257.

[46] G. Tzitzéica, Sur une nouvelle classes de surfaces, C.R. Acad. Sci. Paris 150 (1910) 955, 1227.

[47] R.K. Dodd and R.K. Bullough, Polynomial Conserved Densities for the sine-Gordon Equations, Proc. Roy. Soc. Lond. A 352 (1977) 481 [INSPIRE].

[48] A.V. Mikhailov, Integrability ofa two-dimensional generalization of the Toda chain, JETP Lett. 30 (1979) 414.

[49] V.E. Zakharov and A.B. Shabat, A scheme for integrating the nonlinear equations of mathematical physics by the method of the inverse scattering problem, Funct. Anal. Appl. 8 (1974) 226. 
[50] M.J. Ablowitz, D.J. Kaup, A.C. Newell and H. Segur, Method For Solving The Sine-Gordon Equation, Phys. Rev. Lett. 30 (1973) 1262 [InSPIRE].

[51] M.J. Ablowitz, D.J. Kaup, A.C. Newell and H. Segur, Nonlinear-Evolution Equations of Physical Significance, Phys. Rev. Lett. 31 (1973) 125 [InSPIRE].

[52] A.M. Polyakov, Quark Confinement and Topology of Gauge Groups, Nucl. Phys. B 120 (1977) 429 [INSPIRE].

[53] R. Daviet and N. Dupuis, Nonperturbative functional renormalization-group approach to the sine-Gordon model and the Lukyanov-Zamolodchikov conjecture, Phys. Rev. Lett. 122 (2019) 155301 [arXiv: 1812.01908] [INSPIRE].

[54] S. Samuel, The Grand Partition Function in Field Theory with Applications to sine-Gordon, Phys. Rev. D 18 (1978) 1916 [InSPIRE].

[55] F. Buijnsters, A. Fasolino and M. Katsnelson, Motion of domain walls and the dynamics of kinks in the magnetic peierls potential, Phys. Rev. Lett. 113 (2014) 217202.

[56] P. Minnhagen, A. Rosengren and G. Grinstein, Screening properties of a classical two-dimensional coulomb gas from the sine-gordon equation, Phys. Rev. B 18 (1978) 1356.

[57] A.A. Boris, A. Rydh, T. Golod, H. Motzkau, A.M. Klushin and V.M. Krasnov, Evidence for nonlocal electrodynamics in planar josephson junctions, Phys. Rev. Lett. 111 (2013) 117002.

[58] S.R. Coleman, The Quantum sine-Gordon Equation as the Massive Thirring Model, Phys. Rev. D 11 (1975) 2088 [INSPIRE].

[59] S. Mandelstam, Soliton Operators for the Quantized sine-Gordon Equation, Phys. Rev. D 11 (1975) 3026 [INSPIRE].

[60] P. Minnhagen, New renormalization equations for the kosterlitz-thouless transition, Phys. Rev. B 32 (1985) 3088.

[61] A. Luther, Eigenvalue spectrum of interacting massive fermions in one-dimension, Phys. Rev. B 14 (1976) 2153 [inSPIRE].

[62] R. Sasaki and R.K. Bullough, Geometric Theory of Local and Nonlocal Conservation Laws for the sine-Gordon Equation, Proc. Roy. Soc. Lond. A 376 (1981) 401 [InSPIRE].

[63] S.Y. Lou, Abundant symmetries for the (1+1)-dimensional classical Liouville field theory, J. Math. Phys. 35 (1994) 2336 [INSPIRE].

[64] S.Y. Lou, Soliton molecules and asymmetric solitons in three fifth order systems via velocity resonance, J. Phys. Comm. 4 (2020) 041002.

[65] S.Y. Lou, Symmetries of the kadomtsev-petviashvili equation, J. Phys. A 26 (1993) 4387.

[66] S.Y. Lou, Symmetry algebras of the potential Nizhnik-Novikov-Veselov model, J. Math. Phys. 35 (1994) 1755.

[67] S.Y. Lou, Symmetries and algebras of the integrable dispersive long wave equations in (2+1)-dimensional spaces, J. Phys. A 27 (1994) 3235.

[68] S.Y. Lou and X.-M. Qian, Generalized symmetries and algebras of the two-dimensional differential-difference toda equation, J. Phys. A 27 (1994) L641.

[69] V.E. Zakharov, A.V. Odesskii, M. Cisternino and M. Onorato, Five-wave classical scattering matrix and integrable equations, Theor. Math. Phys. 180 (2014) 759.

[70] J.D. Finley, III and J.F. Plebanski, The Classification of All H Spaces Admitting a Killing Vector, J. Math. Phys. 20 (1979) 1938 [InSPIRE]. 
[71] M. Mineev-WEinstein, P.B. Wiegmann and A. Zabrodin, Integrable structure of interface dynamics, Phys. Rev. Lett. 84 (2000) 5106 [nlin/0001007] [INSPIRE].

[72] Q.H. Park, Extended conformal symmetries in real heavens, Phys. Lett. B 236 (1990) 423.

[73] M. Mañas and L.M. Aloson, A hodograph transformation which applies to the Boyer-Finley equation, Phys. Lett. A 320 (2004) 383.

[74] C.P. Boyer and J.D. Finley, III, Killing Vectors in Selfdual, Euclidean Einstein Spaces, J. Math. Phys. 23 (1982) 1126 [InSPIRE].

[75] R.S. Ward, Einstein-Weyl spaces and SU(infinity) Toda fields, Class. Quant. Grav. 7 (1990) L95 [INSPIRE].

[76] S.V. Manakov and P.M. Santini, The dispersionless 2d toda equation: dressing, cauchy problem, longtime behaviour, implicit solutions and wave breaking, J. Phys. A 42 (2009) 095203.

[77] G. Darboux, Lecons Sur la théorie générale des Surfaces. II, Gauthier-Villars, Paris, France (1888).

[78] D. Levi and P. Winternitz, Symmetries and conditional symmetries of differential difference equations, J. Math. Phys. 34 (1993) 3713 [InSPIRE].

[79] C. Cao, X. Geng and Y. Wu, From the special 2+1 toda lattice to the kadomtsev-petviashvili equation, J. Phys. A 32 (1999) 8059.

[80] H.-W. Tam, X.-B. Hu and X.-M. Qian, Remarks on several 2+1 dimensional lattices, J. Math. Phys. 43 (2002) 1008.

[81] J.F. Plebanski, Some solutions of complex Einstein equations, J. Math. Phys. 16 (1975) 2395 [INSPIRE].

[82] S. Manakov and P. Santini, Inverse scattering problem for vector fields and the cauchy problem for the heavenly equation, Phys. Lett. A 359 (2006) 613.

[83] S.V. Manakov and P.M. Santini, On the solutions of the second heavenly and pavlov equations, J. Phys. A 42 (2009) 404013.

[84] B.G. Konopelchenko, W.K. Schief and A. Szereszewski, Self-dual Einstein spaces and the general heavenly equation. Eigenfunctions as coordinates, Class. Quant. Grav. 38 (2021) 045007 [arXiv: 2008.07261] [INSPIRE].

[85] M. Dunajski and L.J. Mason, HyperKähler hierarchies and their twistor theory, Commun. Math. Phys. 213 (2000) 641 [math/0001008] [INSPIRE].

[86] M. Dunajski and L.J. Mason, Twistor theory of hyperKähler metrics with hidden symmetries, J. Math. Phys. 44 (2003) 3430 [math/0301171] [InSPIRE].

[87] F. Neyzi, Y. Nutku and M.B. Sheftel, Multi-Hamiltonian structure of Plebanski's second heavenly equation, J. Phys. A 38 (2005) 8473 [nlin/0505030] [INSPIRE].

[88] A.P. Fordy and J. Gibbons, Integrable Nonlinear Klein-Gordon Equations And Toda Lattices, Commun. Math. Phys. 77 (1980) 21 [inSPIRE].

[89] K. Pohlmeyer, Integrable Hamiltonian Systems and Interactions Through Quadratic Constraints, Commun. Math. Phys. 46 (1976) 207 [INSPIRE].

[90] F. Lund and T. Regge, Unified approach to strings and vortices with soliton solutions, Phys. Rev. D 14 (1976) 1524. 
[91] B.S. Getmanov, New Lorentz-invariant system with exact multisoliton solutions, JETP Lett. 25 (1977) 119.

[92] V.E. Zakharov and A.V. Mikhailov, Relativistically Invariant Two-Dimensional Models in Field Theory Integrable by the Inverse Problem Technique (in Russian), Sov. Phys. JETP 47 (1978) 1017 [INSPIRE].

[93] A.V. Mikhailov, Integrability of the two-dimensional Thirring model, JETP Lett. 23 (1976) 320.

[94] R.S. Ward, Ansatze for Selfdual Yang-Mills Fields, Commun. Math. Phys. 80 (1981) 563 [INSPIRE].

[95] B. Doubrov, E.V. Ferapontov, B. Kruglikov and V.S. Novikov, On a class of integrable systems of Monge-Ampère type, J. Math. Phys. 58 (2017) 063508 [arXiv:1701.02270] [INSPIRE]. 